\title{
Correction to: Neural and dopaminergic correlates of fatigue in Parkinson's disease
}

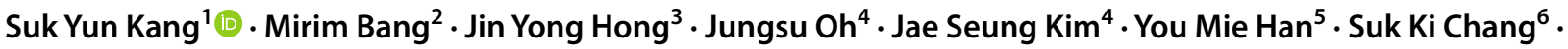 \\ Seun Ah Lee ${ }^{6}$. Uicheul Yoon ${ }^{7}$. Na-Young Shin ${ }^{2}$
}

Published online: 28 January 2020

(c) Springer-Verlag GmbH Austria, part of Springer Nature 2020

Correction to: Journal of Neural Transmission https://doi.org/10.1007/s00702-019-02130-9

The original version of this article unfortunately contained a mistake. The co-author name was incorrect. The correct name should be Jin Yong Hong

Publisher's Note Springer Nature remains neutral with regard to jurisdictional claims in published maps and institutional affiliations

The original article can be found online at https://doi.org/10.1007/ s00702-019-02130-9.

Suk Yun Kang sukyunkang@hanmail.net

$\triangle$ Na-Young Shin nyshin@catholic.ac.kr

1 Department of Neurology, Dongtan Sacred Heart Hospital, Hallym University College of Medicine, 7, Keunjaebong-gil, Hwaseong, Gyeonggi-do 18450, Republic of Korea

2 Department of Radiology, College of Medicine, The Catholic University of Korea, 222 Banpo-daero, Seocho-gu, Seoul 06591, Republic of Korea

3 Department of Neurology, Yonsei University Wonju College of Medicine, Wonju, Republic of Korea
4 Department of Nuclear Medicine, Asan Medical Center, University of Ulsan College of Medicine, Seoul, Republic of Korea

5 Department of Nuclear Medicine, Dongtan Sacred Heart Hospital, College of Medicine, Hallym University, Hwaseong, Republic of Korea

6 Department of Radiology, Hallym University Dongtan Sacred Heart Hospital, Hwaseong, Republic of Korea

7 Department of Biomedical Engineering, College of Health and Medical Science, Catholic University of Daegu, Gyeongsan, Republic of Korea 